Pacific Journal of Mathematics

ON SINGULAR PERTURBATIONS OF SECOND ORDER 


\title{
ON SINGULAR PERTURBATIONS OF SECOND ORDER CAUCHY PROBLEMS
}

\author{
Klaus-J. ENGEL
}

\begin{abstract}
We give an explicit formula for the solution of complete second order Cauchy problems in Banach spaces. Using this formula we derive an estimate for the growth of the solution in terms of an associated scalar ODE. Finally these results are applied to singular perturbations of second order Cauchy problems.
\end{abstract}

1. Introduction. We are concerned with the second order Cauchy problem

$\left(\mathrm{ACP}_{\varepsilon}\right)$

$$
\begin{aligned}
& \varepsilon u_{\varepsilon}^{\prime \prime}(t)+2 B u_{\varepsilon}^{\prime}(t)=A u_{\varepsilon}(t), \quad t \geq 0, \\
& u_{\varepsilon}(0)=u_{0} \in D(A), \quad u_{\varepsilon}^{\prime}(0)=u_{1} \in D(A)
\end{aligned}
$$

in a Banach space $E$ where $A$ is the generator of a strongly continuous cosine family $\left(C_{A}(t)\right)$ commuting with the bounded operator $B \in$ $\mathscr{L}(E)$. It is well known that for $\varepsilon>0\left(\mathrm{ACP}_{\varepsilon}\right)$ is well-posed, i.e., it admits a unique solution which depends continuously on the initial conditions $u_{0}$ and $u_{1}$.

This paper is organized as follows. We first give (in case $\varepsilon=1$ ) an explicit representation of the solution $u(\cdot)$ of $\left(\mathrm{ACP}_{1}\right)$ in terms of $C_{A}(t)$ and $B$. Then we use this formula to derive an estimate for the growth of $u(t)$. In fact, we associate with $\left(\mathrm{ACP}_{1}\right)$ a scalar ODE and show that its solution dominates $\|u(t)\|$. Finally these results are used to show convergence of $u_{\varepsilon}(\cdot)$ as $\varepsilon \downarrow 0$ to the unique solution of $\left(\mathrm{ACP}_{0}\right)$

$$
\begin{aligned}
2 B u_{0}^{\prime}(t) & =A u_{0}(t), \quad t \geq 0, \\
u_{0}(0) & =u_{0}
\end{aligned}
$$

provided that the spectral bound of $-B$ is less than zero. Moreover, from the proof of this result we conclude that under the above assumptions $A B^{-1}$ generates an analytic semigroup.

2. The explicit formula. In order to state the main result of this section we need the following definitions. For a bounded operator $Q \in \mathscr{L}(E)$ we define the modified Bessel function of order zero by

$$
I_{0}(Q):=\sum_{n=0}^{\infty} \frac{\left(\frac{Q}{2}\right)^{2 n}}{(n !)^{2}} \text {. }
$$


Moreover, for two functions $F$ and $G$ defined on $\mathbb{R}_{+}$we denote by $F * G$ the convolution of $F$ and $G$, i.e., $F * G(t):=\int_{0}^{t} F(s) G(t-s) d s$. Using this notation we can show the following result. All integrals are understood in the strong operator topology.

THEOREM 1. Let $A$ be the generator of a strongly continuous cosine family $\left(C_{A}(t)\right)$ which commutes with $B \in \mathscr{L}(E)$. Then the unique solution of the well-posed second order Cauchy problem

$$
\begin{aligned}
& u^{\prime \prime}(t)+2 B u^{\prime}(t)=A u(t), \quad t \geq 0, \\
& u(0)=u_{0} \in D(A), \quad u^{\prime}(0)=u_{1} \in D(A)
\end{aligned}
$$

is given by

$$
u(t)=M_{A, B}(t) u_{0}+N_{A, B}(t) u_{1} .
$$

Here

$$
\begin{aligned}
N_{A, B}(t) & :=e^{-t B} \cdot S_{A+B^{2}}(t), \\
M_{A, B}(t) & :=e^{-t B} \cdot\left(C_{A}(t)+B \cdot S_{A+B^{2}}(t)+B^{2} \cdot C_{A} * S_{A+B^{2}}(t)\right),
\end{aligned}
$$

where

$$
S_{A+B^{2}}(t):=\int_{0}^{t} I_{0}(2 B \sqrt{s(t-s)}) \cdot C_{A}(2 s-t) d s .
$$

Proof. ${ }^{1}$ By [10, Thm. 6] (or see [6, Chap. $\left.2.7 \& 2.8\right]$ ) (1) is wellposed. First we show that $\left(S_{A+B^{2}}(t)\right)$ is the sine family generated by $A+B^{2}$. For this it suffices to verify that the Laplace transform

$$
\mathscr{L}\left(S_{A+B^{2}}\right)(\lambda):=\int_{0}^{\infty} e^{-\lambda t} \cdot S_{A+B^{2}}(t) d t
$$

satisfies the equality

$$
\mathscr{L}\left(S_{A+B^{2}}\right)(\lambda)=R\left(\lambda^{2}, A+B^{2}\right)=\sum_{0}^{\infty} B^{2 n} R\left(\lambda^{2}, A\right)^{n+1}
$$

for $\lambda$ sufficiently large. Indeed,

$$
\begin{aligned}
\mathscr{L}\left(S_{A+B^{2}}\right)(\lambda) & =\int_{0}^{\infty} e^{-\lambda t} \int_{0}^{t} \sum_{0}^{\infty} B^{2 n} \frac{s^{n}}{n !} \frac{(t-s)^{n}}{n !} \cdot C_{A}(2 s-t) d s d t \\
& =\sum_{0}^{\infty} B^{2 n} \int_{0}^{\infty} e^{-\lambda t} \int_{0}^{t} \frac{s^{n}}{n !} \frac{(t-s)^{n}}{n !} \cdot C_{A}(2 s-t) d s d t
\end{aligned}
$$

and (2) follows from the convolution theorem for the Laplace transform and the following lemma.

\footnotetext{
${ }^{1}$ For similar arguments see [1, Thm. 1].
} 
LEMMA 2. If $S_{A}^{*(n+1)}(t)$ denotes the $(n+1)$-fold convolution of the sine family $\left(S_{A}(t)\right)$ generated by $A$, then

$$
\int_{0}^{t} \frac{s^{n}}{n !} \frac{(t-s)^{n}}{n !} \cdot C_{A}(2 s-t) d s=S_{A}^{*(n+1)}(t) .
$$

Proof of Lemma 2. The case $n=0$ is trivial. The general case is obtained by induction from the following computation.

$$
\begin{aligned}
(n !)^{2} & S_{A}^{*(n+1)}(t)=n^{2}((n-1) !)^{2} \int_{0}^{t} S_{A}^{* n}(u) \cdot S_{A}(t-u) d u \\
& =n^{2} \int_{0}^{t} \int_{0}^{u} s^{n-1}(u-s)^{n-1} \cdot C_{A}(2 s-u) d s S_{A}(t-u) d u \\
& =\frac{n^{2}}{2} \int_{0}^{t} \int_{0}^{u} s^{n-1}(u-s)^{n-1}\left(S_{A}(t-2 s)+S_{A}(t+2 s-2 u)\right) d s d u \\
& =n^{2} \int_{0}^{t} \int_{0}^{u} s^{n-1}(u-s)^{n-1} \cdot S_{A}(t-2 s) d s d u \\
& =n^{2} \int_{0}^{t} s^{n-1} \cdot S_{A}(t-2 s) \int_{s}^{t}(u-s)^{n-1} d u d s \\
& =n \int_{0}^{t} s^{n-1}(t-s)^{n} \cdot S_{A}(t-2 s) d s \\
& =\frac{1}{2} \int_{0}^{t} n\left(s^{n-1}(t-s)^{n}-s^{n}(t-s)^{n-1}\right) \cdot S_{A}(t-2 s) d s \\
& =\frac{1}{2}\left[s^{n}(t-s)^{n} \cdot S_{A}(t-2 s)\right]_{s=0}^{s=t}+\int_{0}^{t} s^{n}(t-s)^{n} \cdot C_{A}(t-2 s) d s \\
& =\int_{0}^{t} s^{n}(t-s)^{n} \cdot C_{A}(2 s-t) d s .
\end{aligned}
$$

This completes the proof of Lemma 2.

We proceed with the proof of Theorem 1 and show that

$$
C_{A+B^{2}}(t):=C_{A}(t)+B^{2} \cdot C_{A} * S_{A+B^{2}}(t)
$$

is the cosine family generated by $A+B^{2}$. Again it is sufficient to verify that the Laplace transform of $C_{A+B^{2}}(\cdot)$ satisfies

$$
\mathscr{L}\left(C_{A+B^{2}}\right)(\lambda)=\lambda R\left(\lambda^{2}, A+B^{2}\right)
$$

for $\lambda$ sufficiently large. In fact, by the convolution theorem and the resolvent equation we have

$$
\begin{aligned}
\mathscr{L}\left(C_{A+B^{2}}\right)(\lambda) & =\lambda R\left(\lambda^{2}, A\right)+B^{2} \lambda R\left(\lambda^{2}, A\right) R\left(\lambda^{2}, A+B^{2}\right) \\
& =\lambda R\left(\lambda^{2}, A+B^{2}\right) .
\end{aligned}
$$


Let $x, y \in D(A)$, then the above results imply

$$
\begin{aligned}
& \left(S_{A+B^{2}}(t) x\right)^{\prime \prime}-\left(A+B^{2}\right)\left(S_{A+B^{2}}(t) x\right)=0, \\
& S_{A+B^{2}}(0) x=0, \quad\left(S_{A+B^{2}}(\cdot) x\right)^{\prime}(0)=x, \\
& \left(C_{A+B^{2}}(t) y\right)^{\prime \prime}-\left(A+B^{2}\right)\left(C_{A+B^{2}}(t) y\right)=0, \\
& C_{A+B^{2}}(0) y=y, \quad\left(C_{A+B^{2}}(\cdot) y\right)^{\prime}(0)=0
\end{aligned}
$$

for all $t \geq 0$. Using this one easily verifies (or see [10, Proof of Thm. 6]) that for $N_{A, B}(t):=e^{-t B} \cdot S_{A+B^{2}}(t)$ and $\widetilde{M}_{A, B}(t):=e^{-t B} \cdot C_{A+B^{2}}(t)$ we have

$$
\begin{aligned}
& \left(N_{A, B}(t) x\right)^{\prime \prime}+2 B\left(N_{A, B}(t) x\right)^{\prime}-A\left(N_{A, B}(t) x\right)=0, \\
& N(0) x=0, \quad(N(\cdot) x)^{\prime}(0)=x, \\
& \left(\widetilde{M}_{A, B}(t) y\right)^{\prime \prime}+2 B\left(\widetilde{M}_{A, B}(t) y\right)^{\prime}-A\left(\widetilde{M}_{A, B}(t) y\right)=0, \\
& \widetilde{M}(0) y=y, \quad(\widetilde{M}(\cdot) y)^{\prime}(0)=-B y
\end{aligned}
$$

for all $t \geq 0$. Hence the solution of (1) is given by

$$
\widetilde{M}_{A, B}(t) u_{0}+N_{A, B}(t) u_{1}+N_{A, B}(t) B u_{0}=M_{A, B}(t) u_{0}+N_{A, B}(t) u_{1},
$$

where $M_{A, B}(t)=e^{-t B} \cdot\left(C_{A}(t)+B \cdot S_{A+B^{2}}(t)+B^{2} \cdot C_{A} * S_{A+B^{2}}(t)\right)$.

3. Asymptotics. In this section we estimate the growth of the solution of (1) in terms of an associated scalar ODE (see (3) below). For this purpose we first have to find an estimate for the growth of $\left\|I_{0}(t Q)\right\|$ for a bounded operator $Q \in \mathscr{L}(E)$ and all $t \geq 0$. By definition of the Bessel function it is clear that $\left\|I_{0}(t Q)\right\| \leq I_{0}(t\|Q\|)$ which turns out to be a very rough result. The following lemma relates the Bessel function to the exponential function for which very sharp estimates exist. In fact it is well known (see, e.g., [9, A-III.1]) that for $Q \in \mathscr{L}(E)$ the spectral bound

$$
s(Q):=\sup \{\operatorname{Re} \lambda: \lambda \in \sigma(Q)\}
$$

and the growth bound

$$
\begin{aligned}
\omega(Q):=\inf \left\{\omega \in \mathbb{R}: \text { there exists } M_{\omega}\right. \\
\text { such that } \left.\left\|e^{t Q_{\|}}\right\| \leq M_{\omega} \cdot e^{t \omega} \text { for } t \geq 0\right\}
\end{aligned}
$$

coincide. Combining these results will give a significant improvement of the above estimate.

Lemma 3. Let $Q \in \mathscr{L}(E)$. Then

$$
I_{0}(Q)=\frac{1}{2 \pi} \int_{-\pi}^{\pi} e^{Q \cdot \cos s} d s
$$


Proof. As in the scalar case (see [14, Chap. II, 2.21]) the lemma can be proved by expanding $e^{Q \cos s}$ into a Taylor series and using the fact that for all $n \in \mathbb{N}$

$$
\begin{aligned}
\int_{-\pi}^{\pi}(\cos s)^{2 n+1} d s & =0 \\
\int_{-\pi}^{\pi}(\cos s)^{2 n} d s & =2 \pi \frac{1 \cdot 3 \cdot \cdots \cdot(2 n-1)}{2 \cdot 4 \cdot \cdots \cdot 2 n} .
\end{aligned}
$$

The formula then follows without difficulty.

Before we can state the main result of this section we need some further notations. For $\alpha \in \mathbb{R}_{+}$we denote by

$$
c_{\alpha}(t):=\cosh (\sqrt{\alpha} t) \text { and } s_{\alpha}(t):=1 * c_{\alpha}(t)
$$

the cosine and the sine family, respectively, generated by $\alpha$. Then by [13, Thm. 2.5] (or see [3, Chap. II.5]) there exist constants $M_{A} \geq 1$, $\omega \geq 0$ such that

$$
\left\|C_{A}(t)\right\| \leq M_{A} \cdot c_{\omega}(t) \text { for all } t \geq 0 .
$$

Moreover, for $b<-S(-B)$ there is a constant $M_{B} \geq 1$ such that

$$
\left\|e^{-t B}\right\| \leq M_{B} \cdot e^{-t b} \text { for all } t \geq 0 \text {. }
$$

Now consider the scalar ODE

$$
v^{\prime \prime}(t)+2|b| v^{\prime}(t)=\sqrt{\omega} v(t), \quad v(0)=v_{0}, v^{\prime}(0)=v_{1} .
$$

By Theorem 1 the solution of (3) is given by

$$
v(t)=m_{\omega, b}(t) v_{0}+n_{\omega, b}(t) v_{1},
$$

where

$$
\begin{aligned}
n_{\omega, b}(t) & :=e^{-t|b|} \cdot s_{\omega+b^{2}}(t) \quad \text { and } \\
m_{\omega, b}(t) & :=e^{-t|b|} \cdot\left(c_{\omega}(t)+|b| \cdot s_{\omega+b^{2}}(t)+|b|^{2} \cdot c_{\omega} * s_{\omega+b^{2}}(t)\right) .
\end{aligned}
$$

The following result relates the growth of the solution of (1) to the solution of (3).

THEOREM 4. Let $\left\|e^{-t B}\right\| \leq M_{B} \cdot e^{-t b}$ and $\left\|C_{A}(t)\right\| \leq M_{A} \cdot c_{\omega}(t)$ for all $t \geq 0$. Then for the solution families $\left(N_{A, B}(t)\right)$ and $\left(M_{A, B}(t)\right)$ of (1) the following estimates hold.

(a) If $b \neq 0$, then

$$
\begin{aligned}
\left\|N_{A, B}(t)\right\| & \leq M_{A} M_{B} \cdot e^{t(|b|-b)} n_{\omega, b}(t), \\
\left\|M_{A, B}(t)\right\| & \leq M_{A} M_{B} \max \left\{1, \frac{\|B\|}{|b|}, \frac{\left\|B^{2}\right\|}{|b|^{2}}\right\} \cdot e^{t(|b|-b)} m_{\omega, b}(t),
\end{aligned}
$$

where $n_{\omega, b}(t)$ and $m_{\omega, b}(t)$ are defined as in (4). 
(b) If $b=0$, then

$$
\begin{aligned}
\left\|N_{A, B}(t)\right\| & \leq M_{A} M_{B} \cdot s_{\omega}(t), \\
\left\|M_{A, B}(t)\right\| & \leq M_{A} M_{B} \cdot\left(c_{\omega}(t)+\|B\| \cdot s_{\omega}(t)+\left\|B^{2}\right\| \cdot \frac{t^{2}}{2} s_{\omega}(t)\right) .
\end{aligned}
$$

Proof. (a) By Theorem 1 and Lemma 3 we obtain

$$
\begin{aligned}
N_{A, B}(t) & =e^{-t B} \int_{0}^{t} I_{0}(2 B \sqrt{s(t-s)}) \cdot C_{A}(2 s-t) d s \\
& =\int_{0}^{t} \frac{1}{2 \pi} \int_{-\pi}^{\pi} e^{B(2 \sqrt{s(t-s)} \cos (r)-t)} d r C_{A}(2 s-t) d s .
\end{aligned}
$$

Hence

$$
\begin{aligned}
\left\|N_{A, B}(t)\right\| & \leq M_{A} M_{B} \int_{0}^{t} \frac{1}{2 \pi} \int_{-\pi}^{\pi} e^{b(2 \sqrt{s(t-s)} \cos (r)-t)} d r c_{\omega}(2 s-t) d s \\
& =M_{A} M_{B} \cdot e^{t(|b|-b)} n_{\omega, b}(t)
\end{aligned}
$$

where we used the fact that $2 \sqrt{s(t-s)} \cos (r)-t \leq 0$ for all $s \in[0, t]$ and $r \in[-\pi, \pi]$. Using similar arguments we obtain

$$
\begin{aligned}
\left\|M_{A, B}(t)\right\| \leq & M_{A} M_{B} \cdot e^{-t b}\left(c_{\omega}(t)+\frac{\|B\|}{|b|}|b| \cdot s_{\omega+b^{2}}(t)\right. \\
& \left.+\frac{\left\|B^{2}\right\|}{|b|^{2}}|b|^{2} \cdot c_{\omega} * s_{\omega+b^{2}}(t)\right) \\
\leq & M_{A} M_{B} \max \left\{1, \frac{\|B\|}{|b|}, \frac{\left\|B^{2}\right\|}{|b|^{2}}\right\} \\
& \cdot e^{-t b}\left(c_{\omega}(t)+|b| \cdot s_{\omega+b^{2}}(t)+|b|^{2} \cdot c_{\omega} * s_{\omega+b^{2}}(t)\right) \\
= & M_{A} M_{B} \max \left\{1, \frac{\|B\|}{|b|}, \frac{\left\|B^{2}\right\|}{|b|^{2}}\right\} \cdot e^{t(|b|-b)} m_{\omega, b}(t) .
\end{aligned}
$$

(b) If $b=0$ we conclude from Lemma 3 that $\left\|I_{0}(s B)\right\| \leq M_{B}$; hence

$$
\begin{aligned}
\left\|N_{A, B}(t)\right\| & \leq M_{A} M_{B} \cdot s_{\omega}(t) \quad \text { and } \\
\left\|M_{A, B}(t)\right\| & \leq M_{A} M_{B} \cdot\left(c_{\omega}(t)+\|B\| \cdot s_{\omega}(t)+\left\|B^{2}\right\| \cdot c_{\omega} * s_{\omega}(t)\right) \\
& =M_{A} M_{B} \cdot\left(c_{\omega}(t)+\|B\| \cdot s_{\omega}(t)+\left\|B^{2}\right\| \cdot \frac{t^{2}}{2} s_{\omega}(t)\right) .
\end{aligned}
$$

From Theorem 4 we easily derive the following result. 
Corollary 5. Let $\left\|e^{-t B}\right\| \leq M_{B} \cdot e^{-t b}$ where $b>0$ and $\left\|C_{A}(t)\right\| \leq$ $M_{A} \cdot c_{\omega}(t)$ for all $t \geq 0$. Then the following estimates for the solution families $\left(M_{A, B}(t)\right)$ and $\left(N_{A, B}(t)\right)$ of $(1)$ hold.

$$
\begin{aligned}
\left\|N_{A, B}(t)\right\| & \leq \frac{M_{A} M_{B}}{2 \sqrt{\omega+b^{2}}} \cdot e^{t\left(-b+\sqrt{\omega+b^{2}}\right)} \\
\left\|M_{A, B}(t)\right\| & \leq M_{A} M_{B} \max \left\{1, \frac{\|B\|}{b}, \frac{\left\|B^{2}\right\|}{b^{2}}\right\} \cdot e^{t\left(-b+\sqrt{\omega+b^{2}}\right)} .
\end{aligned}
$$

4. Singular perturbations. There is a substantial literature on singular perturbation problems involving strongly continuous semigroups or cosine families. For details we refer to $[3,4,5]$ and the references therein.

Here we consider singular perturbation problems of the type $\left(\mathrm{ACP}_{\varepsilon}\right)$

$$
\begin{aligned}
& \varepsilon u_{\varepsilon}^{\prime \prime}(t)+2 B u_{\varepsilon}^{\prime}(t)=A u_{\varepsilon}(t), \quad t \geq 0, \\
& u_{\varepsilon}(0)=u_{0} \in D(A), \quad u_{\varepsilon}^{\prime}(0)=u_{1} \in D(A),
\end{aligned}
$$

where it is assumed that $A$ generates a strongly continuous cosine family on some Banach space $E$. While in various papers (e.g. [4, 7, 12]) convergence of the solution $u_{\varepsilon}(\cdot)$ to the solution $u_{0}(\cdot)$ of

$$
2 B u_{0}^{\prime}(t)=A u_{0}(t), \quad t \geq 0, u_{0}(0)=u_{0}
$$

is shown only for $2 B=\operatorname{Id}$ or $B=b>0$ and $A$ the square of a group generator we extend these results to the following situation.

THEOREM 6. Let $A$ be the generator of a strongly continuous cosine family $\left(C_{A}(t)\right)$ on $E$ and $B \in \mathscr{L}(E)$ a bounded operator which commutes with $\left(C_{A}(t)\right)$ and satisfies $s(-B)<0$. Then the unique solution of the second order Cauchy problem $\left(\mathrm{ACP}_{\varepsilon}\right)$ converges as $\varepsilon \downarrow 0$ to the unique solution of the well-posed first order Cauchy problem

$$
\left(\mathrm{ACP}_{0}\right) \quad 2 B u_{0}^{\prime}(t)=A u_{0}(t), \quad t \geq 0, u_{0}(0)=u_{0} \text {. }
$$

Moreover, if $u_{0} \in D\left(A^{2}\right),-b \in(s(-B), 0)$ and

$$
\left\|e^{-t B}\right\| \leq M_{B} \cdot e^{-t b} \text { and }\left\|C_{A}(t)\right\| \leq M_{A} \cdot c_{\omega}(t)
$$

for all $t \geq 0$, then

$$
\begin{aligned}
\left\|u_{0}(t)-u_{\varepsilon}(t)\right\| \leq & \varepsilon \cdot \frac{M_{A} M_{B}}{2 b} \\
\cdot e^{\omega_{0} t} & \left(\left\|A_{0} u_{0}\right\|+M_{A} M_{B}\right. \\
& \left.\cdot \max \left\{1, \frac{\|B\|}{b}, \frac{\left\|B^{2}\right\|}{b^{2}}\right\} t\left\|A_{0}^{2} u_{0}\right\|+\left\|u_{1}\right\|\right),
\end{aligned}
$$

where $\omega_{0}:=\frac{\omega}{2 b}, A_{0}:=\frac{1}{2} A B^{-1}$. 
Proof. As shown in Theorem 1 the Cauchy problem $\left(\mathrm{ACP}_{\varepsilon}\right)$ is well posed. We proceed in several steps. First we show that the solution family $\left(N_{\varepsilon}(t)\right)$ of $\left(\mathrm{ACP}_{\varepsilon}\right)$ converges to zero as $\varepsilon \downarrow 0$. Here and in the sequel we use the simplified notation

$$
N_{\varepsilon}(t):=N_{\frac{A}{\varepsilon}, \frac{B}{\varepsilon}}(t) \quad \text { and } \quad M_{\varepsilon}(t):=M_{\frac{A}{\varepsilon}, \frac{B}{\varepsilon}}(t) .
$$

If $-b \in(s(-B), 0)$, then there exists $M_{B} \geq 1$ such that $\left\|e^{-t B}\right\| \leq$ $M_{B} \cdot e^{-t b}$ for all $t \geq 0$. Let $\left\|C_{A}(t)\right\| \leq M_{A} \cdot c_{\omega}(t)$ for $t \geq 0$; then by Corollary 5

$$
\left\|N_{\varepsilon}(t)\right\| \leq \varepsilon \cdot \frac{M_{A} M_{B}}{2 \sqrt{\varepsilon \omega+b^{2}}} \cdot e^{t \frac{1}{\varepsilon}\left(-b+\sqrt{\varepsilon \omega+b^{2}}\right)} .
$$

Since $-b<0$ an easy calculation shows that

$$
\frac{1}{\varepsilon}\left(-b+\sqrt{\varepsilon \omega+b^{2}}\right) \leq \frac{\omega}{2 b}:=\omega_{0} \text { for all } \varepsilon>0 .
$$

Hence (5) implies

$$
\left\|N_{\varepsilon}(t)\right\| \leq \varepsilon \cdot \frac{M_{A} M_{B}}{2 b} \cdot e^{t \omega_{0}} \quad \text { for all } \varepsilon>0
$$

In the next step we show that $A_{0}:=\frac{1}{2} A B^{-1}$ generates a strongly continuous semigroup $\left(M_{0}(t)\right)$ which turns out to be the limit of $\left(M_{\varepsilon}(t)\right)$ as $\varepsilon \downarrow 0$. As above we obtain from Corollary 5 and (6) the estimate

$$
\left\|M_{\varepsilon}(t)\right\| \leq M_{A} M_{B} \max \left\{1, \frac{\|B\|}{b}, \frac{\left\|B^{2}\right\|}{b^{2}}\right\} \cdot e^{\omega_{0} t} .
$$

Put $C:=M_{A} M_{B} \max \left\{1, \frac{\|B\|}{b}, \frac{\left\|B^{2}\right\|}{b^{2}}, \frac{1}{2 b}\right\}$. Then by (7), (8)

$$
\left\|N_{\varepsilon}(t)\right\| \leq \varepsilon \cdot C \cdot e^{\omega_{0} t} \quad \text { and } \quad\left\|M_{\varepsilon}(t)\right\| \leq C \cdot e^{\omega_{0} t} .
$$

Therefore the Laplace transforms

$$
Q_{\varepsilon}(\lambda):=\mathscr{L}\left(N_{\varepsilon}(\cdot)\right)(\lambda) \quad \text { and } \quad R_{\varepsilon}(\lambda):=\mathscr{L}\left(M_{\varepsilon}(\cdot)\right)(\lambda)
$$

exist for all $\lambda$ with $\operatorname{Re} \lambda>\omega_{0}$ and satisfy the estimates

$$
\begin{aligned}
&\left\|Q_{\varepsilon}(\lambda)\right\| \leq \frac{\varepsilon \cdot C}{\operatorname{Re} \lambda-\omega_{0}} \text { and } \\
&\left\|R_{\varepsilon}(\lambda)\right\| \leq \frac{C}{\operatorname{Re} \lambda-\omega_{0}} \quad \text { for all } \varepsilon>0, \operatorname{Re} \lambda>\omega_{0} .
\end{aligned}
$$


By [8, (9), (11)] we have for $\operatorname{Re} \lambda>\omega_{0}$ and $\varepsilon>0$

$$
\begin{aligned}
& Q_{\varepsilon}(\lambda)=\varepsilon \cdot\left(\varepsilon \lambda^{2}+2 \lambda B-A\right)^{-1} \quad \text { and } \\
& R_{\varepsilon}(\lambda)=(\varepsilon \lambda+2 B)\left(\varepsilon \lambda^{2}+2 \lambda B-A\right)^{-1} .
\end{aligned}
$$

Now from (10), (11) it follows that for fixed $\lambda, \operatorname{Re} \lambda>\omega_{0}$, the family

$$
P_{\varepsilon}(\lambda):=\left(\varepsilon \lambda^{2}+2 \lambda B-A\right)^{-1}
$$

is bounded for $\varepsilon>0$. Moreover, the resolvent equation applied to $P_{\varepsilon}(\lambda)=R\left(\varepsilon \lambda^{2}, A-2 \lambda B\right)$ implies that $\left(P_{\varepsilon}(\lambda)\right)_{\varepsilon>0}$ is a Cauchy net for $\varepsilon \downarrow 0$ and the limit is readily identified as $(2 \lambda B-A)^{-1}$. Again by the resolvent equation it follows that $R_{\varepsilon}(\lambda)$ converges as $\varepsilon \downarrow 0$ to

$$
2 B(2 \lambda B-A)^{-1}=R\left(\lambda, \frac{1}{2} A B^{-1}\right):=R\left(\lambda, A_{0}\right),
$$

where the convergence is uniform for $\lambda$ in compact subsets of $H:=$ $\left\{\lambda \in \mathbb{C}: \operatorname{Re} \lambda>\omega_{0}\right\}$.

In order to show that $A_{0}=\frac{1}{2} A B^{-1}$ generates a strongly continuous semigroup $\left(M_{0}(t)\right)$ it suffices to show the Hille-Yosida estimates

$$
\left\|\frac{d^{n}}{d \lambda^{n}} R\left(\lambda, A_{0}\right)\right\| \leq \frac{C \cdot n !}{\left(\operatorname{Re} \lambda-\omega_{0}\right)^{n+1}} \quad \text { for all } \lambda \in H \text { and } n \in \mathbb{N}_{0} \text {. }
$$

First observe that $R_{\varepsilon}(\cdot)$ (see $\left.[8, \S 3]\right)$ and $R\left(\cdot, A_{0}\right)$ are analytic in $H$. Since $R_{\varepsilon}(\cdot)$ converges as $\varepsilon \downarrow 0$ to $R\left(\cdot, A_{0}\right)$ uniformly on compact subsets of $H$ the Weierstraß convergence theorem implies that

$$
\frac{d^{n}}{d \lambda^{n}} R_{\varepsilon}(\cdot) \rightarrow \frac{d^{n}}{d \lambda^{n}} R\left(\cdot, A_{0}\right) \quad \text { for } \varepsilon \rightarrow 0
$$

uniformly on compact subsets of $H$. Let $M_{\varepsilon, n}(t):=(-1)^{n} t^{n} M_{\varepsilon}(t)$. Then for $\operatorname{Re} \lambda>\omega_{0}$ and $n \in \mathbb{N}_{0}$

$$
\frac{d^{n}}{d \lambda^{n}} R_{\varepsilon}(\lambda)=\mathscr{L}\left(M_{\varepsilon, n}(\cdot)\right)(\lambda)
$$

and we conclude from (9) that

$$
\left\|\frac{d^{n}}{d \lambda^{n}} R_{\varepsilon}(\lambda)\right\| \leq \frac{C \cdot n !}{\left(\operatorname{Re} \lambda-\omega_{0}\right)^{n+1}} .
$$

Combining this with (13) yields the desired estimate (12). This shows that $A_{0}=\frac{1}{2} A B^{-1}$ generates a strongly continuous semigroup $\left(M_{0}(t)\right)$. Hence the Cauchy problem $\left(\mathrm{ACP}_{0}\right)$ is well posed.

In the last step we show that $M_{\varepsilon}(t)$ converges to $M_{0}(t)$ uniformly for $t$ in bounded subsets of $\mathbb{R}_{+}$. In order to estimate $M_{0}(t)-M_{\varepsilon}(t)$ we need the identity

$$
\begin{gathered}
\left(M_{0}(t)-M_{\varepsilon}(t)\right) f=M_{0} *\left(M_{\varepsilon}-\frac{2}{\varepsilon} B \cdot N_{\varepsilon}\right)(t) A_{0} f \\
=M_{0} * N_{\varepsilon}^{\prime}(t) A_{0} f \text { for all } f \in D\left(A_{0}\right)=D(A)
\end{gathered}
$$


To verify (14) note that the Laplace transform of $M_{0}(\cdot)$ gives the resolvent $R\left(\cdot, A_{0}\right)$. Using this, (11), the convolution and the uniqueness theorem for the Laplace transform we obtain (14). Integrating by parts the right hand side of (14) yields

$$
\begin{aligned}
& \left(M_{0}(t)-M_{\varepsilon}(t)\right) f \\
& \quad=N_{\varepsilon}(t) A_{0} f+M_{0} * N_{\varepsilon}(t) A_{0}^{2} f \text { for all } f \in D\left(A_{0}^{2}\right)=D\left(A^{2}\right) .
\end{aligned}
$$

Hence for $f \in D\left(A^{2}\right)$ we deduce from (7), (8)

$$
\begin{aligned}
& \left\|\left(M_{0}(t)-M_{\varepsilon}(t)\right) f\right\| \\
& \leq \varepsilon \cdot \frac{M_{A} M_{B}}{2 b} \cdot m_{0}(t)\left\|A_{0} f\right\| \\
& +\varepsilon \cdot \frac{\left(M_{A} M_{B}\right)^{2}}{2 b} \max \left\{1, \frac{\|B\|}{b}, \frac{\left\|B^{2}\right\|}{b^{2}}\right\} \cdot m_{0} * m_{0}(t)\left\|A_{0}^{2} f\right\| \\
& \leq \varepsilon \cdot \frac{M_{A} M_{B}}{2 b} \cdot m_{0}(t)\left(\left\|A_{0} f\right\|+M_{A} M_{B}\right. \\
& \left.\quad \cdot \max \left\{1, \frac{\|B\|}{b}, \frac{\left\|B^{2}\right\|}{b^{2}}\right\} t\left\|A_{0}^{2} f\right\|\right),
\end{aligned}
$$

where $m_{0}(t):=e^{\omega_{0} t}=e^{\frac{\omega}{2 b} t}$. To estimate $u_{0}(t)-u_{\varepsilon}(t)$ note that

$$
u_{0}(t)=M_{0}(t) u_{0}, \quad u_{\varepsilon}(t)=M_{\varepsilon}(t) u_{0}+N_{\varepsilon}(t) u_{1} .
$$

Accordingly, from (7), (15) we obtain for all initial values $u_{0} \in D\left(A^{2}\right)$, $u_{1} \in D(A)$

$$
\begin{aligned}
\left\|u_{0}(t)-u_{\varepsilon}(t)\right\| \leq \varepsilon & \cdot \frac{M_{A} M_{B}}{2 b} \\
\cdot e^{\omega_{0} t} & \left(\left\|A_{0} u_{0}\right\|+M_{A} M_{B}\right. \\
& \left.\cdot \max \left\{1, \frac{\|B\|}{b}, \frac{\left\|B^{2}\right\|}{b^{2}}\right\} t\left\|A_{0}^{2} u_{0}\right\|+\left\|u_{1}\right\|\right) .
\end{aligned}
$$

Since the operator families $\left(M_{0}(t)-M_{\varepsilon}(t)\right)_{\varepsilon>0}$ and $\left(N_{\varepsilon}(t)\right)_{\varepsilon>0}$ are uniformly bounded for $t$ in bounded subsets of $\mathbb{R}_{+}$we finally conclude that $u_{0}(t)-u_{\varepsilon}(t)$ converges to zero as $\varepsilon \downarrow 0$ uniformly for $t$ in bounded intervals for all initial values $u_{0}, u_{1} \in D(A)$.

Using Theorem 6 it is also possible to obtain results on the convergence of the derivatives of $u_{\varepsilon}(\cdot)$. In fact, if in $\left(\mathrm{ACP}_{\varepsilon}\right)$ it is assumed that $u_{0} \in D\left(A^{2}\right)$, then $v_{\varepsilon}(\cdot):=u_{\varepsilon}^{\prime}(\cdot)$ is twice differentiable and solves 
the Cauchy problem

$$
\begin{aligned}
& \varepsilon v_{\varepsilon}^{\prime \prime}(t)+2 B v_{\varepsilon}^{\prime}(t)=A v_{\varepsilon}(t), \quad t \geq 0, \\
& v_{\varepsilon}(0)=u_{1}, \quad v_{\varepsilon}^{\prime}(0)=\frac{1}{\varepsilon}\left(A u_{0}-2 B u_{1}\right) .
\end{aligned}
$$

On the other hand the solution $u_{0}(\cdot)$ of $\left(\mathrm{ACP}_{0}\right)$ is twice differentiable as well and $v_{0}(\cdot):=u_{0}^{\prime}(\cdot)$ is the solution of

$$
2 B v_{0}^{\prime}(t)=A v_{0}(t), \quad t \geq 0, \quad v_{0}(0)=\frac{1}{2} A B^{-1} u_{0} .
$$

Hence, by Theorem 1, Theorem 6

$$
v_{0}(t)-v_{\varepsilon}(t)=M_{0}(t) \frac{1}{2} A B^{-1} u_{0}-M_{\varepsilon}(t) u_{1}+\frac{1}{\varepsilon} N_{\varepsilon}(t)\left(A u_{0}-2 B u_{1}\right) \text {. }
$$

In particular, for $\frac{1}{2} A B^{-1} u_{0}=u_{1}$ we obtain the following result.

COROLlary 7. Let the assumptions of Theorem 6 hold. In addition assume that $\frac{1}{2} A B^{-1} u_{0}=u_{1}$. Then the derivatives $u_{\varepsilon}^{\prime}(\cdot)$ converge uniformly on bounded intervals of $\mathbb{R}_{+}$to $u_{0}^{\prime}(\cdot)$ as $\varepsilon \downarrow 0$. Moreover, if $u_{1} \in D\left(A^{2}\right)$, then

$$
\begin{aligned}
\left\|v_{0}^{\prime}(t)-v_{\varepsilon}^{\prime}(t)\right\| \leq & \varepsilon \cdot \frac{M_{A} M_{B}}{2 b} \\
\cdot e^{\omega_{0} t} & \left(\left\|A_{0} u_{1}\right\|+M_{A} M_{B}\right. \\
& \left.\cdot \max \left\{1, \frac{\|B\|}{b}, \frac{\left\|B^{2}\right\|}{b^{2}}\right\} t\left\|A_{0}^{2} u_{1}\right\|\right),
\end{aligned}
$$

where $\omega_{0}:=\frac{\omega}{2 b}, A_{0}:=\frac{1}{2} A B^{-1}$.

From the proof of Theorem 6 we also obtain the following result on multiplicative perturbation.

Corollary 8. Let $A$ be the generator of a strongly continuous cosine family $\left(C_{A}(t)\right)$ on $E$ and $B \in \mathscr{L}(E)$ a bounded operator which commutes with $\left(C_{A}(t)\right)$ and satisfies $s(-B)<0$. Then $A B^{-1}$ generates an analytic semigroup.

Proof. We only have to show that the semigroup generated by $A_{0}:=$ $\frac{1}{2} A B^{-1}$ is analytic. To this end observe that there exists $\alpha=a+i b \in$ $\mathbb{C},|\alpha|=1, a, b>0$ such that the assumptions of Theorem 6 still hold if we replace $B$ by $\alpha B$ and $\bar{\alpha} B$, respectively. Hence we conclude that $\alpha A_{0}$ and $\bar{\alpha} A_{0}$ are generators and the assertion follows from the next result. 
LEMMA 8. Let $\alpha A_{0}$ and $\bar{\alpha} A_{0}$ be generators of strongly continuous semigroups on $E$, where $\alpha=a+i b \in \mathbb{C},|\alpha|=1$ and $a, b>0$. Then $A_{0}$ generates an analytic semigroup.

Proof. We may assume that $\omega\left(\alpha A_{0}\right), \omega\left(\bar{\alpha} A_{0}\right) \leq 0$; otherwise consider $A_{0}-\omega$ instead of $A_{0}$ where $\omega$ is chosen such that $\omega\left(\alpha A_{0}\right)$, $\omega\left(\bar{\alpha} A_{0}\right) \leq \omega a$. Now it is clear that $(\alpha+\bar{\alpha}) A_{0}$, hence $A_{0}$ generates a bounded semigroup and by [11, Chap. 2, Thm. 5.2] it suffices to show that there exists a constant $C>0$ such that for all $\sigma>0, \tau \neq 0$

$$
\left\|R\left(\sigma+i \tau, A_{0}\right)\right\| \leq \frac{C}{|\tau|} .
$$

We consider two cases. First assume $\tau>0$. Since $\bar{\alpha} A_{0}$ is a generator the Hille-Yosida theorem yields a constant $C$ such that

$$
\begin{aligned}
\left\|R\left(\sigma+i \tau, A_{0}\right)\right\| & =\left\|\bar{\alpha} R\left(\bar{\alpha}(\sigma+i \tau), \bar{\alpha} A_{0}\right)\right\| \\
& \leq \frac{C}{a \sigma+b \tau} \leq \frac{C}{b \tau} \text { for all } \tau>0 .
\end{aligned}
$$

For $\tau<0$ we obtain a similar estimate using the fact that $\alpha A_{0}$ is a generator on $E$.

\section{REFERENCES}

[1] K.-J. Engel, An explicit formula for semigroups generated by $2 \times 2$ operator matrices, to appear in Res. Math.

[2] K.-J. Engel and J. A. Goldstein, Singular perturbation, preprint 1990.

[3] H. O. Fattorini, Second Order Linear Differential Equations in Banach Spaces, North-Holland Mathematics Studies 108, North-Holland, 1985.

[4] _ Singular perturbation and boundary layer for an abstract Cauchy problem, J. Math. Anal. Appl., 97 (1983), 529-571.

[5] _ The hyperbolic singular perturbation problem: an operator theoretic approach, J. Differential Equations, 70 (1987), 1-41.

[6] J. A. Goldstein, Semigroups of Linear Operators and Applications, Oxford University Press, New York, 1985.

[7] J. Kisyński, On second order Cauchy's problem in a Banach space, Bull. Acad. Polon. Sci., 18 (1970), 371-374.

[8] I. V. Mel'nikova, A Miranda-Feller-Phillips theorem for a complete second order equation in a Banach space, Soviet Math., 29 (1985), 42-48.

[9] R. Nagel (ed.), One-parameter Semigroups of Positive Operators, Lecture Notes Mathematics 1184, Springer-Verlag, Berlin-Heidelberg-New York-Tokyo, 1986.

[10] F. Neubrander, Well-posedness of higher order abstract Cauchy problems, Trans. Amer. Math. Soc., 295 (1986), 257-290.

[11] A. Pazy, Semigroups of Linear Operators and Applications to Partial Differential Equations, Springer-Verlag, Berlin-Heidelberg-New York-Tokyo, 1986.

[12] A. Y. Schoene, Semigroups and a class of singular perturbation problems, Indiana Univ. Math. J., 20 (1970), 247-263. 
[13] M. Sova, Cosine operator functions, Rozprawy Mat., 49 (1966), 1-47.

[14] G. N. Watson, A Treatise on the Theory of Bessel Functions, Cambridge University Press, Cambridge, 1966.

Received August 2, 1990 and in revised form January 16, 1991.

MATHEMATISChES INSTITUT DER UNIVERSitÄt TÜBINGEN

AUF DER MORGENSTELLE 10

D-7400 TÜBINGEN, GERMANY 



\title{
PACIFIC JOURNAL OF MATHEMATICS EDITORS
}

\author{
V. S. VARADARAJAN \\ (Managing Editor) \\ University of California \\ Los Angeles, CA 90024-1555-05 \\ Herbert Clemens \\ University of Utah \\ Salt Lake City, UT 84112 \\ F. Michael Christ \\ University of California \\ Los Angeles, CA 90024-1555 \\ THOMAS ENRIGHT \\ University of California, San Diego \\ La Jolla, CA 92093
}

Nicholas ERcolani

University of Arizona

Tucson, AZ 85721

R. FINN

Stanford University

Stanford, CA 94305

VAUGHAN F. R. JoNeS

University of California

Berkeley, CA 94720

C. C. MOORE

University of California

Berkeley, CA 94720

MARTIN SchaRLEMANN

University of California

Santa Barbara, CA 93106

HAROLd StaRK

University of California, San Diego

La Jolla, CA 92093

SteVen Kerckhoff

Stanford University

Stanford, CA 94305

\section{ASSOCIATE EDITORS}

\begin{tabular}{|c|c|c|c|c|}
\hline R. ARENS & $\begin{array}{l}\text { E. F. BECKENBACH } \\
(1906-1982)\end{array}$ & Neumann & $\begin{array}{c}\text { F. WoLF } \\
(1904-1989)\end{array}$ & K. YosHIDA \\
\hline \multicolumn{5}{|c|}{ SUPPORTING INSTITUTIONS } \\
\hline \multicolumn{2}{|c|}{ UNIVERSITY OF ARIZONA } & \multicolumn{3}{|c|}{ UNIVERSITY OF OREGON } \\
\hline \multicolumn{2}{|c|}{ UNIVERSITY OF BRITISH COLUMBIA } & \multicolumn{3}{|c|}{ UNIVERSITY OF SOUTHERN CALIFORNI } \\
\hline \multicolumn{2}{|c|}{ CALIFORNIA INSTITUTE OF TECHNOLOGY } & \multirow{2}{*}{\multicolumn{3}{|c|}{ STANFORD UNIVERSITY }} \\
\hline \multicolumn{2}{|c|}{ UNIVERSITY OF CALIFORNIA } & & & UNIVERSITY OF HAWAII \\
\hline \multicolumn{2}{|c|}{ MONTANA STATE UNIVERSITY } & \multicolumn{3}{|c|}{ UNIVERSITY OF TOKYO } \\
\hline \multicolumn{2}{|c|}{ UNIVERSITY OF NEVADA, RENO } & \multicolumn{3}{|c|}{ UNIVERSITY OF UTAH } \\
\hline \multicolumn{2}{|c|}{ NEW MEXICO STATE UNIVERSITY } & \multicolumn{3}{|c|}{ WASHINGTON STATE UNIVERSITY } \\
\hline \multicolumn{2}{|c|}{ OREGON STATE UNIVERSITY } & UNIVER: & OF WASHINC & \\
\hline
\end{tabular}




\section{Pacific Journal of Mathematics}

Vol. 152, No. $1 \quad$ January, 1992

B. V. Rajarama Bhat, On a characterization of velocity maps in the space of observables .......................................

John David Brillhart, Note on the discriminant of certain cyclotomic period

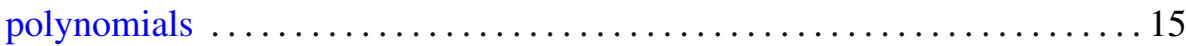

Steven R. Costenoble and Stefan Waner, The equivariant Thom

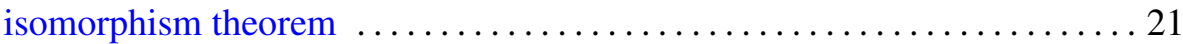

Karl Heinz Dovermann and Dong Youp Suh, Smith equivalence for finite

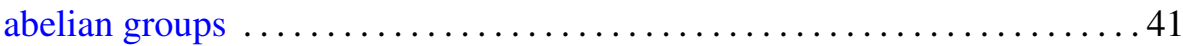

Klaus-Jochen Engel, On singular perturbations of second order Cauchy

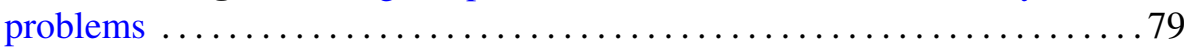

Angel Ferrandez and Pascual Lucas, On surfaces in the 3-dimensional

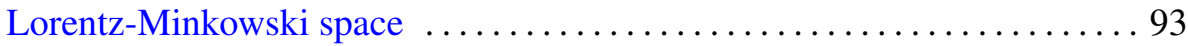

Brian Hartley, A general Brauer-Fowler theorem and centralizers in locally

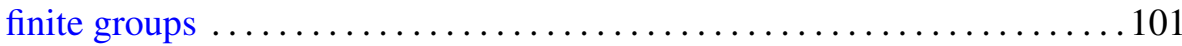

Joel Hass, Intersections of least area surfaces .................... 119

Edward Kissin, Indices of unbounded derivations of $C^{*}$-algebras $\ldots \ldots \ldots 125$

Erhard Luft and Denis Karmen Sjerve, On regular coverings of

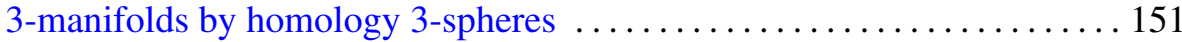

Daniel Pecker, Sur la projection de variétés algébriques réelles .......... 165

Claus Scheiderer, Some remarks on orderings under finite field

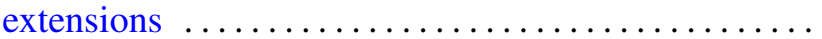

Fernando Serrano, Elliptic surfaces with an ample divisor of genus two 